\title{
Interaction of organic molecules with smectite clay minerals: a review
}

\author{
IAN C. BOURG ${ }^{12 *}$
}

${ }^{1}$ Department of Civil and Environmental Engineering, Princeton University, Princeton, New Jersey 08544, USA (*correspondence: bourg@ princeton.edu)

${ }^{2}$ Princeton Environmental Institute, Princeton University, Princeton, New Jersey 08544, USA

Interactions between organic molecules and clay minerals play a key role in influencing the abundance of soil organic matter and the fate and transport of organic contaminants. Therefore, the sorption of organic solutes on clay minerals has been extensively analyzed over the last several decades. Here, we present a comprehensive review of existing data on the interactions of organic molecules with smectite clay, the predominant clay minerals in soils and sediments formed in temperate weathering environments. Because of the vast amount of existing data, we focus particularly on adsorption at low loadings. We identify key trends associated with atomistic-level interactions (particularly the role of hydrophobic vs. specific interactions, the important of ionizable groups, and sensitivity to the exchangeable cations) and with larger-scale interactions (including sensitivity to the arrangement of clay nanoparticles, the sorption or highmolecular-weight compounds, and the formation of macromolecular assemblages at the clay-water interface). We end with a discussion of implications regarding soil or sediment carbon dynamics and contamiant fate and transport. 